\title{
Pneumokokkenschutzimpfung
}

\section{Risikokinder sicher identifizieren}

\begin{abstract}
Seit Sommer 2010 gibt es eine neue STIKO-Empfehlung, die eine Indikationsimpfung von gefährdeten Kindern im Alter von zwei bis fünf Jahren mit einem 13-valenten Pneumokokken-Konjugatimpfstoff empfiehlt. Aktuell betrifft dies die Jahrgänge 2006 bis 2009. Wie Kinder mit einem entsprechenden Risiko im Praxisalltag erkannt werden können, erklärt der Kinder- und Jugendarzt Dr. Andreas Busse, Tegernsee.
\end{abstract}

? Lassen Sie uns noch einmal kurz Revue passieren: Welche Kinder fallen in die Gruppe der Risikokinder?

Busse: Unter Risikokindern verstehen wir alle Kinder, für die aufgrund von Vorerkrankungen Infektionskrankheiten gefährlicher sind als für andere Kinder und die daher auf besonderen Schutz angewiesen sind. Das sind zum Beispiel Kinder mit chronischen Atemwegserkrankungen - Stichwort Asthma bronchiale und hyperreagibles Bronchialsystem -, mit angeborenen Herzfehlern, mit neurologischen Krankheiten wie Epilepsie oder mit chronischen Nierenerkrankungen. Weiterhin gehören Kinder mit angeborenen Fehlbildungen und Chromosomen-Krankheiten, zum Beispiel mit Down-Syndrom, in die Gruppe der Risikokinder.

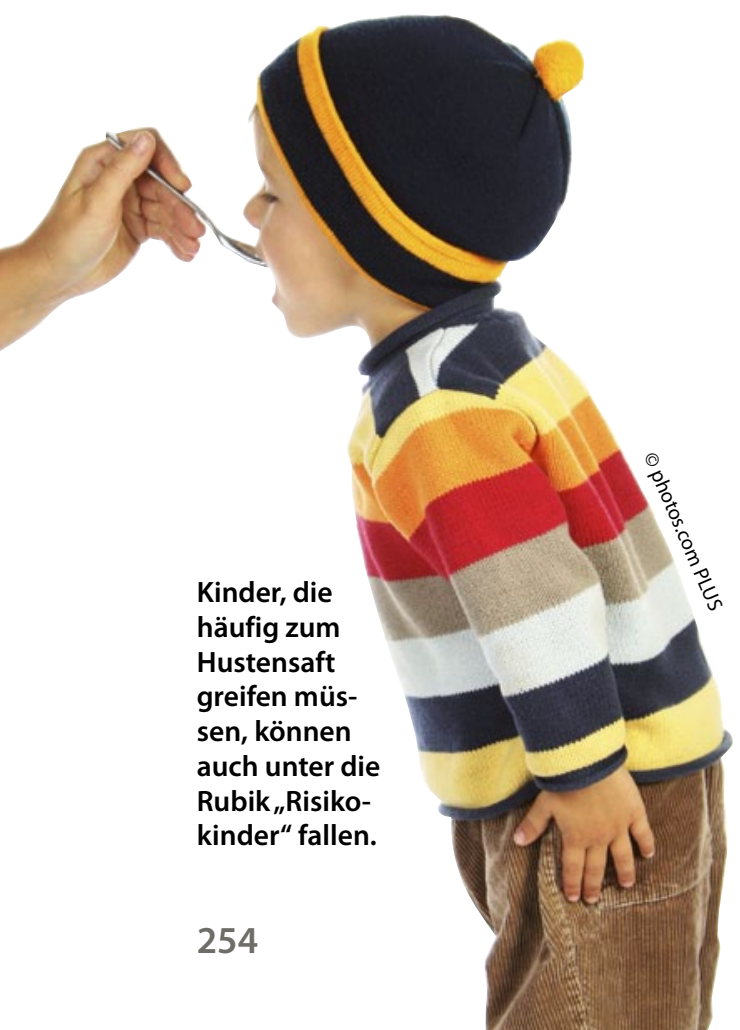

? Gibt es darüber hinaus weitere Impfindikationen, die nicht so explizit in der aktuellen STIKO-Empfehlung genannt sind? Busse: Es gibt sicher Grenzfälle. Überlegen sollte man bei Kindern, die in einer Gemeinschaftseinrichtung betreut werden und früh rezidivierende Atemwegsinfekte haben. Sie können auch unter die Rubrik Risikokinder fallen, obwohl sie nicht die strenge Definition der STIKO erfüllen. Zudem ermöglicht die STIKO-Empfehlung, dass jeder Kinder- und Jugendarzt das individuelle Risiko seiner Patienten prüft und entsprechende Impfempfehlungen außerhalb der Definition ausspricht. Ich mache das bei Patienten, die ständig Atemwegsinfekte haben und in einer Kinderkrippe betreut werden. Mit der Ergänzungsimpfung mit dem 13-valenten Pneumokokken-Impfstoff kann man den Eltern eine Maßnahme anbieten, die dazu beiträgt, dass schwere Mittelohrentzündungen und andere schwere Erkrankungen deutlich seltener auftreten.

? Welches Vorgehen hat sich in der Praxis bewährt, um Risikokinder zu identifizieren?

Busse: Jeder Kinderarzt kennt seine Risikopatienten schon dadurch, dass sie meist sehr viel häufiger als gesunde Kinder in der Praxis erscheinen. In unserer Praxis erkennen wir sie zudem an einer Kennzeichnung auf der Karteikarte, viele Praxis- und EDV-Systeme weisen automatisch darauf hin. Das ist letztendlich auch für die Abrechnung relevant, da für diese Kinder ein Zuschlag über eine besondere Risikoziffer abgerechnet werden kann.

? Welche Rolle spielen die Vorsorgeuntersuchungen, z.B. die recht neu eingeführte

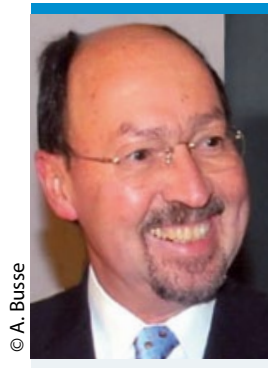

„Die STIKO-Empfehlung ermöglicht, dass jeder Kinder- und Jugendarzt das individuelle Risiko seiner Patienten prüft und entsprechende Impfempfehlungen außerhalb der Definition ausspricht."

Dr. med. Andreas Busse Niedergelassener Pädiater in Tegernsee

U7a, bei der Identifikation von Risikokindern?

Busse: Die neue Vorsorgeuntersuchung U7a hat den Vorteil, dass wir die Patienten zu dem definierten Zeitpunkt - nämlich zwischen dem 34. und 36. Lebensmonat - noch einmal sehen. Das ist eine sehr gute Gelegenheit, erneut zu prüfen, ob für dieses Kind die Ergänzungsimpfung in Frage kommt.

? Warum ist die Compliance der Eltern eines der zentralen Themen, wenn man von Impferfolgen spricht?

Busse: Aus aktuellen Befragungen wissen wir, dass die Aufklärung durch den Arzt bei den Eltern den höchsten Stellwert hat. Auch die „Hardliner" und echten Zweifler sind zu überzeugen, wenn sie ein gutes Verhältnis zu ihrem Kinderarzt haben und ihm vertrauen. Oft werden aber auch Impfhindernisse angenommen, die gar nicht da sind. Dazu gehört beispielsweise, dass ein Kind einen Schnupfen hat. Hierzu sagt die STIKO ganz eindeutig, dass ein banaler Infekt kein Impfhindernis ist. Dann liegt es beim Arzt, den Eltern zu sagen: „Das Kind hat zwar einen Schnupfen, aber wir können trotzdem gefahrlos impfen."

! Herr Dr. Busse, vielen Dank für das Gespräch.

Das Interview führte Birgit Dickoré. 